\title{
Figures of Merit for High-Performance Transparent Electrodes Using Dip-Coated Silver Nanowire Networks
}

\author{
Sergio B. Sepulveda-Mora ${ }^{1}$ and Sylvain G. Cloutier ${ }^{2}$ \\ ${ }^{1}$ Department of Electrical \& Computer Engineering, University of Delaware, Newark, DE 19716, USA \\ ${ }^{2}$ Department of Electrical Engineering, École de Technologie Supérieure, Montréal, QC, Canada H3C 1 K3 \\ Correspondence should be addressed to Sergio B. Sepulveda-Mora, sergiosm@udel.edu
}

Received 15 June 2012; Revised 20 August 2012; Accepted 21 August 2012

Academic Editor: Yanqiu Zhu

Copyright ( 2012 S. B. Sepulveda-Mora and S. G. Cloutier. This is an open access article distributed under the Creative Commons Attribution License, which permits unrestricted use, distribution, and reproduction in any medium, provided the original work is properly cited.

\begin{abstract}
Homogeneous, highly conductive, and transparent silver nanowire thin films were fabricated using a simple dip-coating technique and a subsequent annealing step. Silver nanowires with two different average lengths $(11 \mu \mathrm{m}$ and $19 \mu \mathrm{m})$ were used in the sample preparation to analyze the dependence of the sheet resistance on the length of the one-dimensional nanostructures. The best sample had a sheet resistance of $10.2 \Omega / \square$ with optical transmittance of $89.9 \%$. Two figures of merit, the electrical to optical conductivity ratio $\left(\sigma_{\mathrm{DC}} / \sigma_{\mathrm{OP}}\right)$ and $\phi_{\mathrm{TC}}$, were obtained for all the samples in order to measure their performance as transparent conductive materials.
\end{abstract}

\section{Introduction}

Transparent conductive electrodes (TCE) are unique materials that manifest both high electrical conductivity and high optical transmittance. Such materials are an essential component in optoelectronic devices which require to extract electrons from the active layer while allowing the light to pass through. Some applications of TCE are organic solar cells, thin film solar cells, light-emitting diodes (LEDs), liquid crystal displays (LCDs), touch screens, antistatic coatings, and electromagnetic shielding, among others [1]. Transparent conductive thin films are of special interest in the solar energy industry [2], mainly for their application in the new generation of low-cost photovoltaic devices.

The performance of TCE films can be assessed through two crucial parameters, namely, the sheet resistance $\left(R_{\mathrm{sh}}\right)$ and the optical transmittance $(T)$. It is always desirable to have a low $R_{\mathrm{sh}}$ with a high value of $T$. Nevertheless, there is an inherit relation between these variables. Lower values of $R_{\mathrm{sh}}$ leads to a decrease in the optical transmittance and vice versa.

Metal oxides are currently the most widely used material for TCE. Aluminum-doped Zinc Oxide is used in amorphous silicon solar cells, fluorine doped tin oxide (FTO), and indium-doped tin oxide (ITO) are used in organic solar cells and
LEDs [3]. During the last five decades, ITO has dominated the market as the most commonly used TCE in optoelectronic applications owing to its high optical transparency at very low sheet resistance (typical values are $T=80 \%$ and $R_{\mathrm{sh}}=10 \Omega / \square$ in glass substrate [4]).

Although ITO has demonstrated to work very well in optoelectronic applications in the last years, it also has some drawbacks which are leading to search for alternative materials. First, the fabrication of ITO is costly and complex because of the high vacuum required in the sputtering process. Second, indium is becoming scarcer with the pass of time, which in turn produces an increment in the price of ITO. Finally, ITO is completely unsuitable for flexible devices owing to its brittle nature [5].

Recently, emerging candidates have been proposed as replacement for metal oxides, specially for applications in flexible devices. Random carbon nanotubes (CNTs) networks [6-8] and graphene [9] have been characterized as TCE thin films. In fact, organic solar cells have been successfully implemented and tested using CNTs $[10,11]$ and graphene $[12,13]$ as the front transparent contact. In general, CNTs and graphene have shown stable flexibility but still with inferior performance than that of ITO with regard to $R_{\mathrm{sh}}$ and $T$. 
A new paradigm to achieve both high conductivity and high transparency is to use a mesh of highly conductive metal nanowires covering only a small fraction of a surface. Copper [14] and silver [3, 4, 15-20] nanowire networks have been reported to behave as TCE with comparable performance to ITO and also with bending and stretching stability.

Other approaches include the preparation of TCE thin films using hybrid composite materials such as silver nanowires with metal oxides [21, 22], a mixture of silver and gold nanowires [23], and silver nanowires with polymer [24-26].

Silver nanowire networks stand out among all the other candidates to replace ITO in optoelectronic devices due not only to their electrical and optical characteristics (comparable and in some cases better than ITO [19]) but also because of the constant $R_{\text {sh }}$ while the film is being bended or stretched $[3,15,16,18,20]$. Furthermore, silver nanowire meshes have been proven to effectively replace ITO in organic solar cells [4, 22, 24, 27, 28], sound emitting devices [29], touch screens [17], organic LEDs [25, 26], and electrical conductive adhesives [30] applications.

While different deposition techniques of silver nanowires on rigid and flexible substrates have been already demonstrated $[3,15-17,19,20]$, most of them showed an inferior performance than that of ITO on rigid substrates. In this paper, we report the fabrication of uniform silver nanowire thin films using a straightforward dip-coating technique and a subsequent annealing step. In our best sample, we obtained a very low sheet resistance $\left(R_{\text {sh }}=10.2 \Omega / \square\right)$ with an outstanding optical transmittance $(89.9 \%)$. We fabricated and characterized silver nanowire networks with two different average lengths leading to analyse the influence of nanowire morphology in the overall performance of the film as a transparent conductive electrode. Two figures of merit defined in the literature were used to determine the quality of the films.

\section{Experimental}

Silver nanowires in ethanol solution were purchased from two different companies, namely, Blue nano (product no. SLV-NW-90) and Nanogap (product number NF Ag-3101E). The first one with a concentration of $10 \mathrm{mg} / \mathrm{mL}$ and the second one with $0.87 \mathrm{mg} / \mathrm{mL}$. Further SEM analysis of the morphology showed an average diameter of $103 \mathrm{~nm}$ and average length of $19 \mu \mathrm{m}$ for the Blue nano sample; the average diameter and length for the Nanogap nanowires were $108 \mathrm{~nm}$ and $11 \mu \mathrm{m}$, respectively.

2.1. Sample Preparation. Microscope slides with dimensions $25 \times 75 \times 1.0 \mathrm{~mm}$ were obtained from Fisher Scientific and used as glass substrate. Each slide was carefully divided into two halves with a glass cutter yielding a glass substrate of dimensions $25 \times 37.5 \times 1.0 \mathrm{~mm}$.

The glass slides were thoroughly cleaned with detergent and washed out with deionized water. All of the glass substrate was then treated with acetone and isopropanol baths in the sonicator during $15 \mathrm{~min}$ each.

Silver nanowires in ethanol solutions from both sources were prepared with concentrations of $5 \mathrm{mg} / \mathrm{mL}, 10 \mathrm{mg} / \mathrm{mL}$, and $20 \mathrm{mg} / \mathrm{mL}$. Uniform $25 \times 25 \mathrm{~mm}$ thin films of silver nanowires were fabricated by dip coating the glass slide on the aforementioned solutions. Sticker paper was attached to the rear side of the substrate to prevent the formation of a silver nanowire mesh on the backside of the sample. Different coverage densities were achieved by dip coating several layers of the same solution, allowing a 2 min waiting time between layers to let the ethanol evaporate. After the last layer, the sticker paper was peeled off the glass and the rear side of the sample was carefully cleaned up with ethanol to remove any remaining residues. Then the sample was cut again to form a $25 \times 25 \mathrm{~mm}$ square thin film of silver nanowires on glass substrate as shown in Figure 1(a). The dip coater (Dip Master 50 from Chemat inc.) was set up at a with draw speed of $200 \mathrm{~mm} / \mathrm{min}$ and a still time of $1 \mathrm{~s}$ for all the samples made in this study.

The use of extra material has to be considered when using the dip-coating technique since an undesired layer will be formed in the rear side of the substrate. A lower withdraw speed yields a thinner layer and therefore less wasted material.

After the dip-coating step, the samples were annealed at $250^{\circ} \mathrm{C}$ during $30 \mathrm{~min}$ in a tube furnace to improve the contact between nanowires. A mechanical pump was used to extract the air of the tube furnace in order to prevent oxidation of the samples.

Commercially available ITO and FTO were purchased and characterized for comparison purposes. ITO on glass with dimensions of $25 \times 25 \times 0.7 \mathrm{~mm}$ was acquired from Delta Technologies (product number CG-61IN) with a sheet resistance range of $15-30 \Omega / \square$.

2.2. Characterization. An e-beam evaporator was utilised to place two circular electrode pads of silver on opposite corners of each sample (see Figure 1(a)). The evaporator was set up to deposit the pads with a thickness of $100 \mathrm{~nm}$, and the masks were custom designed to set the diameter of the pads in $3 \mathrm{~mm}$. Then the sheet resistance of the silver nanowire films was determined using a Keithley 236 source measure unit.

The transmittance was measured with a light source coupled through an optical fiber to a silicon-biased detector with a wavelength range of 350-1100 nm. A tungsten halogen lamp (LS-1) from Ocean Optics was employed as the light source. The samples were placed orthogonal to the incident light. Transmittance was calculated as the ratio of the light intensity detected after the sample and the light intensity detected after a glass slide which was used as reference.

The morphology of the silver nanowires was characterized with a scanning electron microscope (SEM) JEOL JSM $7400 \mathrm{~F}$.

Coverage density was calculated from imaging the samples with an optical microscope at 100x magnification. First, 10 images were taken from each sample. Then, the color depth of the images was decreased to 1 bit per pixel. The modified image was read bit by bit, and the coverage density was defined as the ratio of the number of fewer pixels to the total number of pixels contained in the image. The coverage density data presented in Figure 4 is the average of the 10 images. 


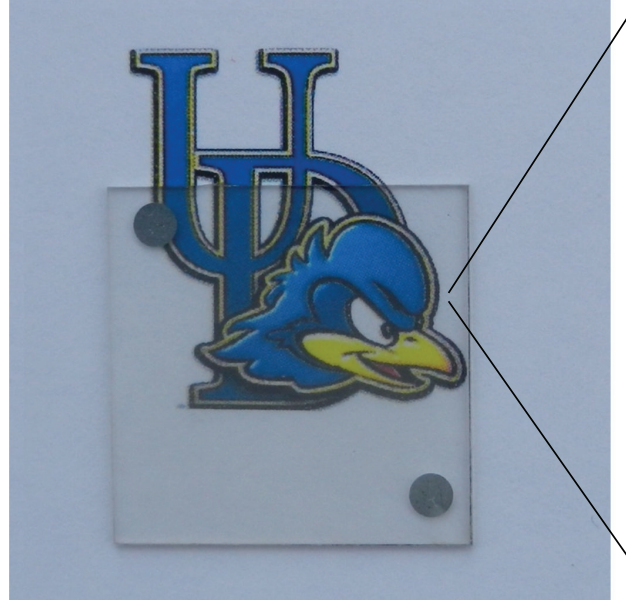

(a)

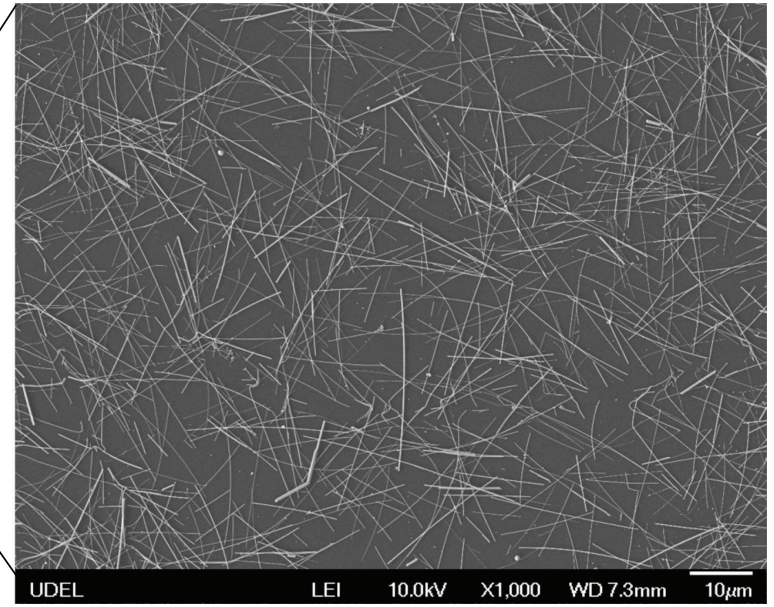

(b)

Figure 1: (a) Photograph of a typical sample with $R_{\mathrm{sh}}=24 \Omega / \square$ and $T=90 \%$ (Nanogap $\langle L\rangle=11 \mu \mathrm{m}$ ) on top of the University of Delaware logo. (b) SEM image of the same sample showing the random distribution of silver nanowires throughout the surface of the glass substrate.

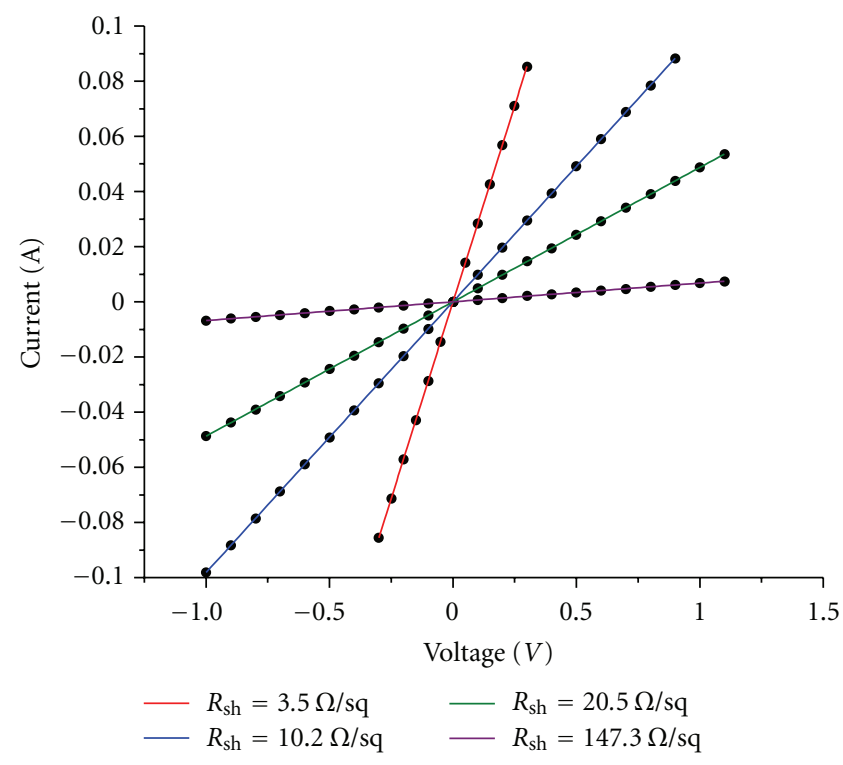

(a)

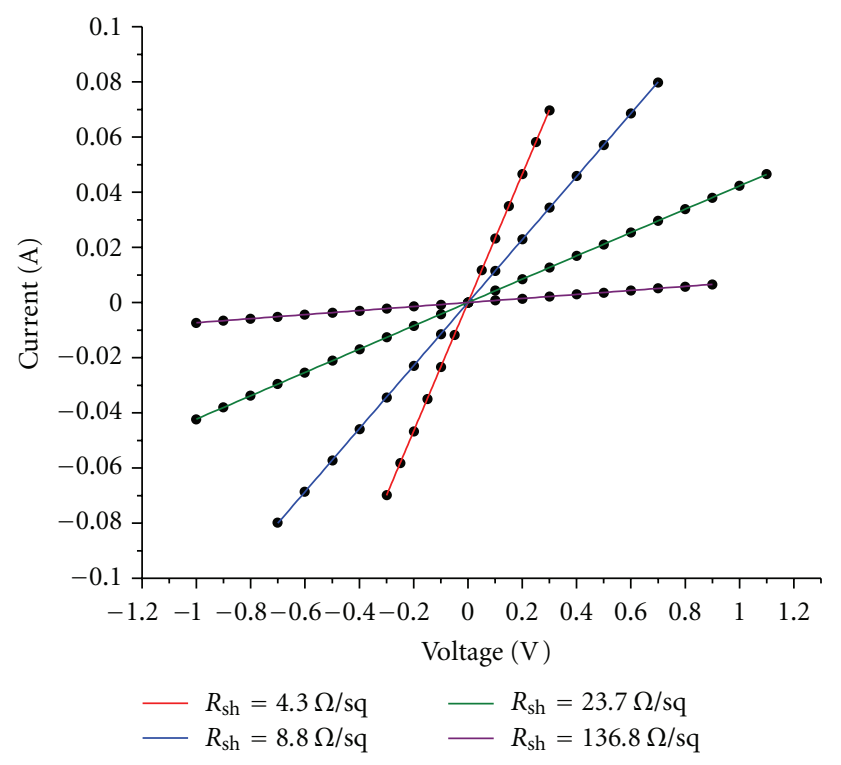

(b)

FiguRE 2: $I$ - $V$ curves of different samples for (a) long nanowires (Blue nano) and (b) short nanowires (Nanogap).

The adhesion properties were measured by using a wellknown test. The AgNW samples did not pass the scotch tape test, and hence encapsulation is required to guarantee a high adhesion of the nanowires to the substrate.

The stability of the samples in ambient conditions was tested by repeating the characterization process after a certain period of time. The samples did not oxidize, and both the electrical and optical properties remained unchanged after 3 months of fabrication.

\section{Results and Discussion}

One can see from Figure 1(a) that the dip-coated samples are highly uniform and transparent, since the logo underneath is clearly visible. The SEM image displayed in Figure 1(b) demonstrates the even distribution of the nanowires throughout the surface of the substrate. Using the dip-coating technique, the one-dimensional structures are placed in random directions creating an arbitrary network where charge carriers have different paths to go through.

The current-voltage characteristics of the samples with different sheet resistance for the long and the short nanowires are shown in Figure 2. The straight lines demonstrate the linear response and the quality of the films as electrodes.

The nanowire-nanowire contact resistance is notably improved by the annealing process. When the silver nanowires mesh undergoes relatively high temperatures, the junction of the nanowires starts to melt increasing the 


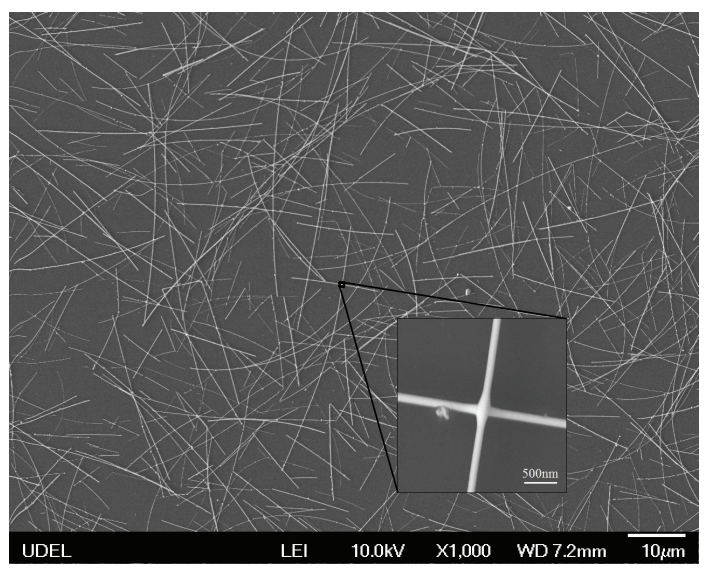

FIGURE 3: SEM image of a silver nanowire network after annealing $\left(250^{\circ} \mathrm{C} \sim 30 \mathrm{~min}\right)$.

conductivity of the film, as shown in the inset of Figure 3. Lee et al. [4] reported a decrease of one order of magnitude on $R_{\mathrm{sh}}$ after heating treatment. We observed similar results in our experiments.

Every transparent conductive material presents a fundamental tradeoff between electrical conductivity and optical transmittance. In order to elucidate this phenomenon, we consider three parameters, the sheet resistance $\left(R_{\mathrm{sh}}\right)$, the optical transmittance $(T)$, and the coverage density $(D)$. Figure 4 shows how these variables are mutually related. As $R_{\mathrm{sh}}$ becomes higher, the values of $T$ are increased as well. Conversely, a higher $D$ produces lower $R_{\mathrm{sh}}$ values.

The plots shown in Figure 4 are the experimental data (filled dots) and a nonlinear fit (smooth lines) obtained using the curve fitting tool available in Matlab. The mathematical model for the long nanowires (Blue nano) is displayed in

$$
\begin{array}{cl}
T=f\left(R_{\mathrm{sh}}\right)=0.96-1.03 R_{\mathrm{sh}}^{-1.18}, & R^{2}=0.99, \\
D=f\left(R_{\mathrm{sh}}\right)=11.78+105.7 R_{\mathrm{sh}}^{-1.46}, & R^{2}=0.95 .
\end{array}
$$

Equations (3) and (4) model the behavior of $T$ and $D$ as a function of $R_{\mathrm{sh}}$ for the short nanowires (Nanogap)

$$
\begin{array}{ll}
T=f\left(R_{\mathrm{sh}}\right)=0.92-16.06 R_{\mathrm{sh}}^{-2.77}, & R^{2}=0.99, \\
D=f\left(R_{\mathrm{sh}}\right)=7.75+24.65 R_{\mathrm{sh}}^{-0.48}, & R^{2}=0.94 .
\end{array}
$$

The values of $R$-squared in all of the previous equations are very close to the unity which indicates a good fit of the model with the experimental data.

Figure 4(a) is a plot of $T$ and $D$ as a function of $R_{\mathrm{sh}}$ for the long nanowires (Blue nano), while Figure 4(b) is the same plot for the short nanowires (Nanogap). The sheet resistance and optical transmittance data for commercial samples of ITO and FTO on glass substrate are also shown for comparison purposes. Both nanowires samples have a superior performance than that of FTO by far. The long nanowires (Blue nano) show better results than ITO, while the short nanowires (Nanogap) have a slight inferior performance in comparison with ITO. This will be further discussed later when we explain the figures of merit.

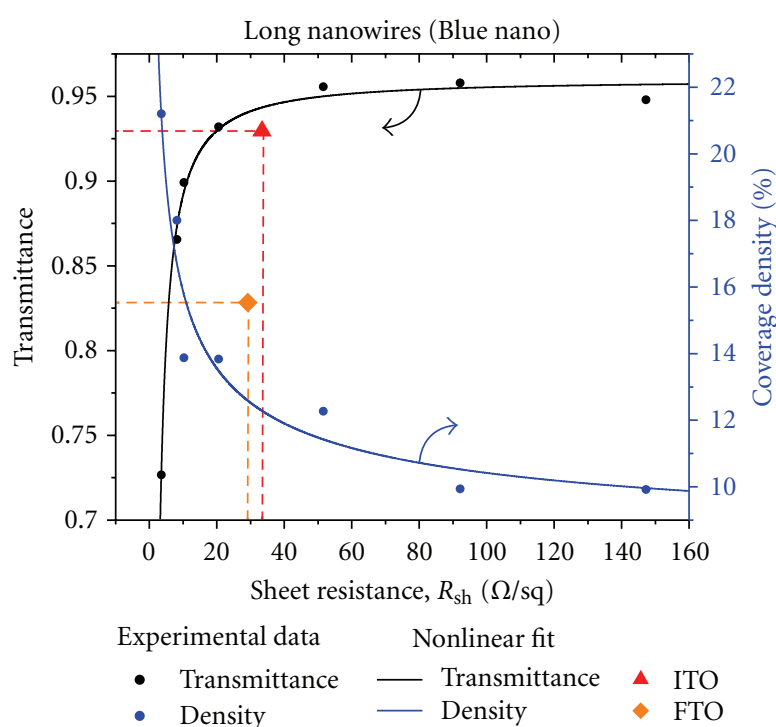

(a)

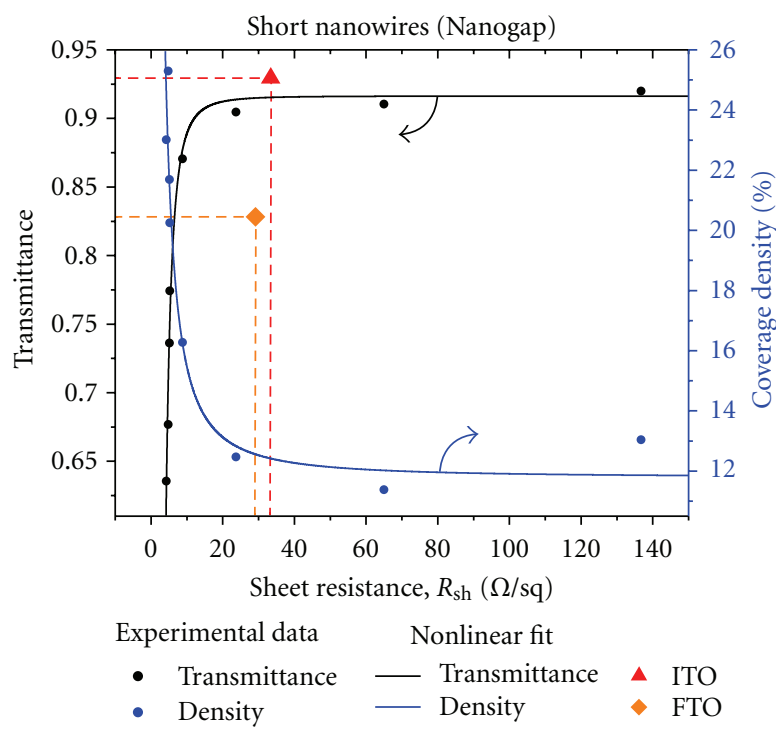

(b)

Figure 4: Optical transmittance and coverage density versus $R_{\mathrm{sh}}$ for (a) Blue nano AgNWs $(\langle L\rangle=11 \mu \mathrm{m})$ and (b) Nanogap AgNWs $(\langle L\rangle=19 \mu \mathrm{m})$.

Different coverage densities are obtained by increasing the number of dip-coated layers deposited on the glass substrate. Higher coverage density values are achieved by increasing the concentration of the silver nanowires in the ethanol solution. The correlation of the three important parameters $\left(R_{\mathrm{sh}}, T\right.$, and $\left.D\right)$ is conspicuously depicted in Figure 5. The microscopy analysis is in good agreement with the data shown in the Figure 4. A smaller empty space between the silver nanowires leads to a higher coverage density and a lower sheet resistance, which in turn results in a lower transmittance.

In order to determine which combinations of $R_{\mathrm{sh}}$ and $T$ are the optimal, we need to use some figures of merit defined 


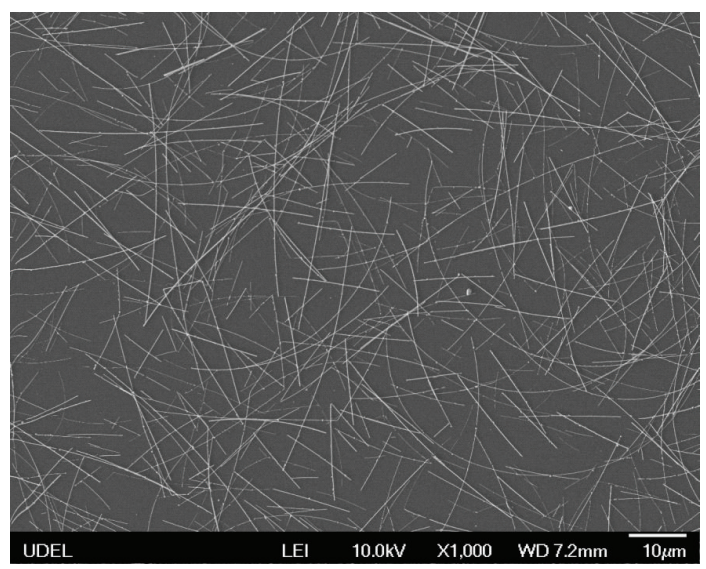

(a)

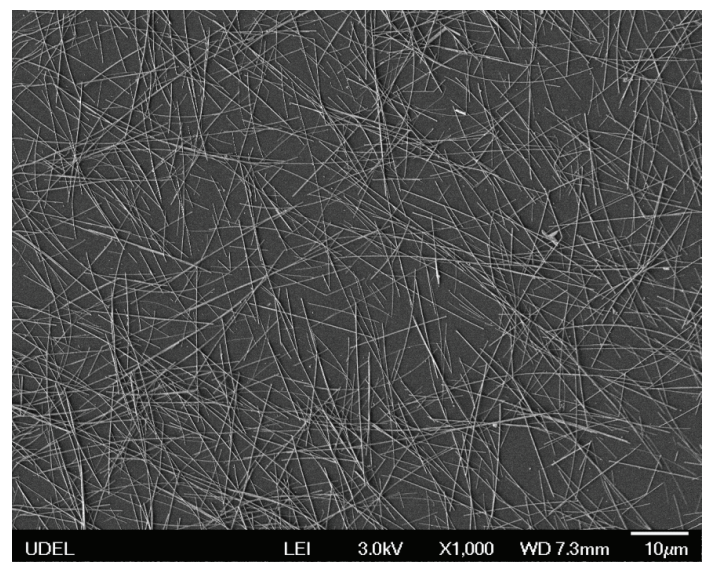

(c)

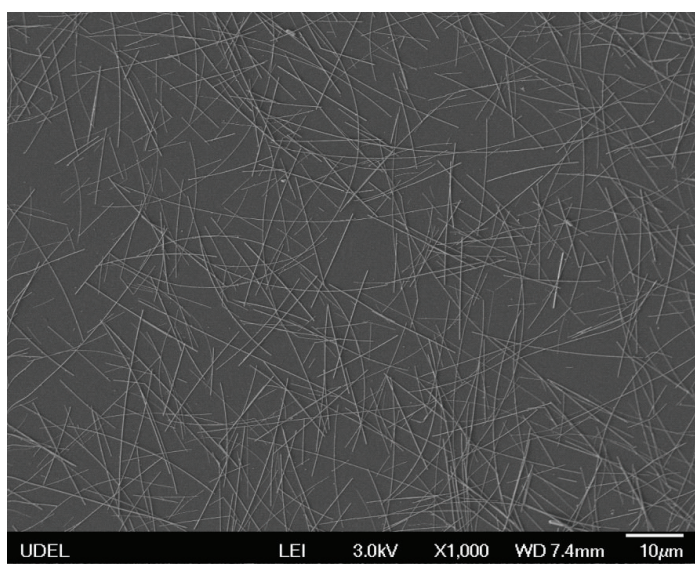

(b)

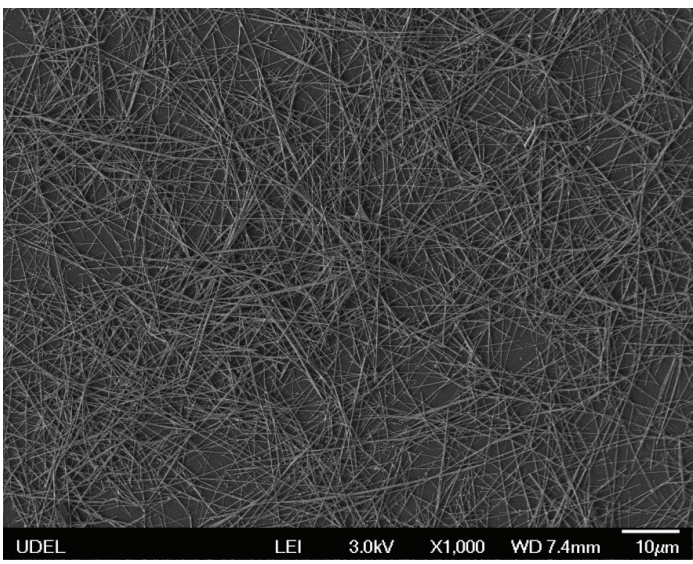

(d)

Figure 5: SEM images of Blue nano AgNWs $(\langle L\rangle=19 \mu \mathrm{m})$ samples with different sheet resistance $\left(R_{\text {sh }}\right)$, optical transmittance $(T)$, and coverage density (D) (a) $R_{\text {sh }}=147 \Omega / \square, T=94.8 \%, D=9.9 \%$. (b) $R_{\text {sh }}=20.5 \Omega / \square, T=93.2 \%, D=13.8 \%$. (c) $R_{\text {sh }}=10.2 \Omega / \square$, $T=89.9 \%, D=13.9 \%$. (d) $R_{\text {sh }}=3.5 \Omega / \square, T=72.7 \%, D=21.2 \%$.

in the literature. The first one is the electrical to optical conductivity ratio used by some authors $[19,20,31]$ :

$$
T=\left(1+\frac{188.5}{R_{\mathrm{sh}}} \cdot \frac{\sigma_{\mathrm{OP}}}{\sigma_{\mathrm{DC}}}\right)^{-2},
$$

or

$$
\frac{\sigma_{\mathrm{DC}}}{\sigma_{\mathrm{OP}}}=\frac{188.5}{R_{\mathrm{sh}} \cdot\left(T^{-1 / 2}-1\right)} .
$$

From (6), we can easily determine the electrical to optical conductivity ratio from the $R_{\mathrm{sh}}$ and $T$ parameters. This figure of merit will help us to judge how well the silver nanowire network behaves as a transparent conductive electrode. The higher the $\sigma_{\mathrm{DC}} / \sigma_{\mathrm{OP}}$, the better the TCE.

We can deduce a model for the electrical to optical conductivity by using the nonlinear fits presented before. If we solve (1) and (3) for $R_{\mathrm{sh}}$ and then replace it on (6), we will obtain the figure of merit $\sigma_{\mathrm{DC}} / \sigma_{\mathrm{OP}}$ as a function of the transmittance $T$ for the long nanowires (7) and for the short ones (8):

$$
\begin{aligned}
& \frac{\sigma_{\mathrm{DC}}}{\sigma_{\mathrm{OP}}}=\frac{188.5}{T^{-1 / 2}-1} \cdot\left(\frac{0.96-T}{1.03}\right)^{1 / 1.18}, \\
& \frac{\sigma_{\mathrm{DC}}}{\sigma_{\mathrm{OP}}}=\frac{188.5}{T^{-1 / 2}-1} \cdot\left(\frac{0.92-T}{16.06}\right)^{1 / 2.77} .
\end{aligned}
$$

Figure 6(a) shows a plot of the electrical to optical conductivity ratio as a function of the optical transmittance for both the long (Blue nano) and the short (Nanogap) silver nanowires. The scattered dots represent the experimental data while the smooth lines depict the plot of (7) and (8).

It turned out that the longer nanowires present a better performance than the short nanowires. The highest values of $\sigma_{\mathrm{DC}} / \sigma_{\mathrm{OP}}$ were $339 \Omega^{-1}$ and $300 \Omega^{-1}$ for the long (Blue nano) and the short (Nanogap) nanowires, respectively; that is, an increase of $73 \%$ in the length of the silver nanowires will yield to an increment of $13 \%$ in the electrical to optical conductivity ratio. 


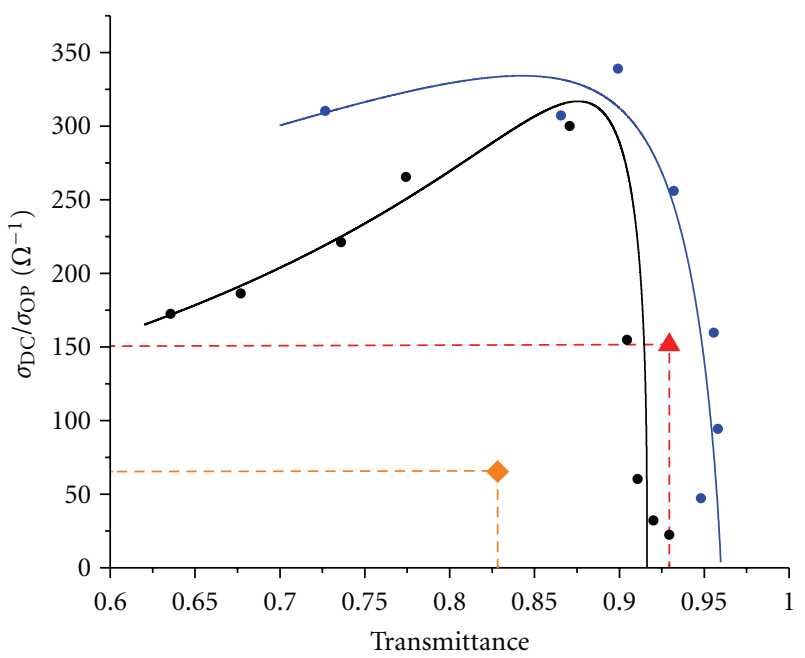

Long nanowires (Blue nano) Short nanowires (Nanogap)

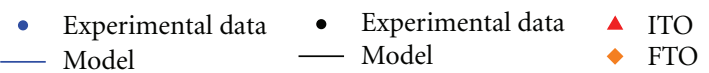

(a)

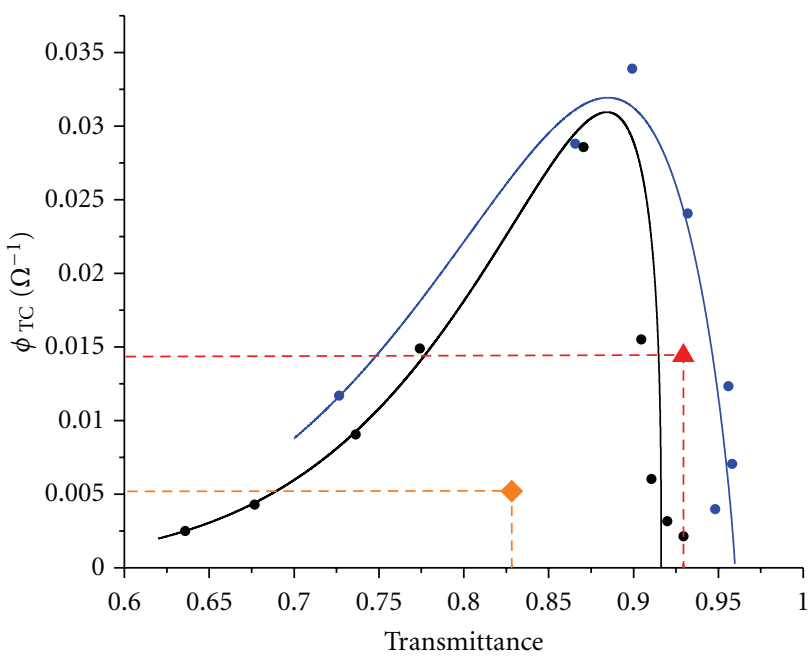

Long nanowires (Blue nano) Short nanowires (Nanogap)

- Experimental data - Experimental data $\Delta$ ITO

- Model $\longrightarrow$ Model $\longrightarrow$ FTO

(b)

FIGURE 6: Figures of merit versus optical transmittance (a) $\sigma_{\mathrm{DC}} / \sigma_{\mathrm{OP}}$ and (b) $\phi_{\mathrm{TC}}$.

Another figure of merit for transparent conductive materials was defined by Haacke [32]:

$$
\phi_{\mathrm{TC}}=\frac{T^{10}}{R_{\mathrm{sh}}} .
$$

Analogously to the procedure we did for the electrical to optical conductivity ratio, we determined a model for the figure of merit $\phi_{\text {TC }}$. This model is described by (10). The model is displayed in Figure 6(b):

$$
\begin{aligned}
& \phi_{\mathrm{TC}}=T^{10} \cdot\left(\frac{0.96-T}{1.03}\right)^{1 / 1.18}, \\
& \phi_{\mathrm{TC}}=T^{10} \cdot\left(\frac{0.92-T}{16.06}\right)^{1 / 2.77} .
\end{aligned}
$$

The superior performance of the long nanowires (Blue nano) is confirmed in Figure $6(\mathrm{~b})$, where $\phi_{\mathrm{TC}}$ is plotted as a function of the optical transmittance for the short and the long silver nanowires. The figures of merit for ITO and FTO are plotted as well. Lee et al. [4] and $\mathrm{Hu}$ et al. [3] reported that longer nanowires will cause a lower $R_{\text {sh }}$ which is in accordance with our results. The long nanowires can interconnect to each other with lower coverage density, allowing a higher transmittance with the same $R_{\text {sh }}$. The highest values of $\phi_{\mathrm{TC}}$ for our samples were $0.034 \Omega^{-1}$ and $0.029 \Omega^{-1}$ for the long and the short nanowires, respectively. These values are better than the ones corresponding to the commercial samples of metal oxides (FTO and ITO). In the same way, an increment of $73 \%$ in the average length of the nanowires (from $11 \mu \mathrm{m}$ to $19 \mu \mathrm{m}$ ) produces a raise in $\phi_{\mathrm{TC}}$ of $17 \%$.

The relative high diameter of the silver nanowires produces a rough surface. This might be an issue for organic devices with typical thickness of $100 \mathrm{~nm}$ or less. In the other hand, the rough surface improves the light trapping in photovoltaic devices, making these electrodes an appealing material for organic solar cell fabrication.

\section{Conclusions}

In summary, we fabricated uniform silver nanowire thin films on glass substrate using a straightforward dip-coating technique. We used short and long silver nanowires, and we demonstrated that longer nanowires lead to a higher performance of transparent conductive electrodes. Our best sample had a $R_{\text {sh }}=10.2 \Omega / \square, T=0.899, D=13.9 \%$, $\sigma_{\mathrm{DC}} / \sigma_{\mathrm{OP}}=339 \Omega^{-1}$, and $\phi_{\mathrm{TC}}=0.034 \Omega^{-1}$. We showed the dependence between sheet resistance, optical transmittance, and coverage density. We made use of two figures of merit to determine the performance of our samples and to compare them with metal oxides. We conclude that random networks of silver nanowires can immediately replace metal oxides such as ITO and FTO in any optoelectronic application owing to their electrical, optical, and mechanical properties which made them suitable for a great variety of devices.

\section{References}

[1] G. Haacke, “Transparent conducting coatings," Annual Review of Materials Science, vol. 7, pp. 73-93, 1977.

[2] C. G. Granqvist, "Transparent conductors as solar energy materials: a panoramic review," Solar Energy Materials and Solar Cells, vol. 91, no. 17, pp. 1529-1598, 2007.

[3] L. Hu, H. S. Kim, J. Y. Lee, P. Peumans, and Y. Cui, "Scalable coating and properties of transparent, flexible, silver nanowire electrodes," ACS Nano, vol. 4, no. 5, pp. 2955-2963, 2010.

[4] J. Y. Lee, S. T. Connor, Y. Cui, and P. Peumans, "Solutionprocessed metal nanowire mesh transparent electrodes," Nano Letters, vol. 8, no. 2, pp. 689-692, 2008. 
[5] Z. Chen, B. Cotterell, and W. Wang, "The fracture of brittle thin films on compliant substrates in flexible displays," Engineering Fracture Mechanics, vol. 69, no. 5, pp. 597-603, 2002.

[6] H. Z. Geng, K. K. Kim, K. P. So, Y. S. Lee, Y. Chang, and H. L. Young, "Effect of acid treatment on carbon nanotube-based flexible transparent conducting films," Journal of the American Chemical Society, vol. 129, no. 25, pp. 7758-7759, 2007.

[7] T. M. Barnes, J. Van De Lagemaat, D. Levi et al., "Optical characterization of highly conductive single-wall carbonnanotube transparent electrodes," Physical Review B, vol. 75, no. 23, Article ID 235410, 2007.

[8] M. H. Andrew Ng, L. T. Hartadi, H. Tan, and C. H. Patrick Poa, "Efficient coating of transparent and conductive carbon nanotube thin films on plastic substrates," Nanotechnology, vol. 19, no. 20, Article ID 205703, 2008.

[9] A. Vollmer, X. L. Feng, X. Wang et al., "Electronic and structural properties of graphene-based transparent and conductive thin film electrodes," Applied Physics A, vol. 94, no. 1, pp. 1-4, 2009.

[10] N. Pimparkar, M. Chowalla, and M. A. Alam, "Device optimization for organic photovoltaics with cnt networks as transparent electrode," in Proceedings of the Photovoltaic Specialists Conference, 2008.

[11] M. A. Contreras, T. Barnes, J. Van De Lagemaat et al., "Replacement of transparent conductive oxides by single-wall carbon nanotubes in $\mathrm{Cu}(\mathrm{In}, \mathrm{Ga}) \mathrm{Se} 2$-based solar cells," Journal of Physical Chemistry C, vol. 111, no. 38, pp. 14045-14048, 2007.

[12] X. Wang, L. Zhi, and K. Müllen, "Transparent, conductive graphene electrodes for dye-sensitized solar cells," Nano Letters, vol. 8, no. 1, pp. 323-327, 2008.

[13] J. Wu, H. A. Becerril, Z. Bao, Z. Liu, Y. Chen, and P. Peumans, "Organic solar cells with solution-processed graphene transparent electrodes," Applied Physics Letters, vol. 92, no. 26, Article ID 263302, 2008.

[14] H. Wu, L. Hu, M. W. Rowell et al., "Electrospun metal nanofiber webs as high-performance transparent electrode," Nano Letters, vol. 10, no. 10, pp. 4242-4248, 2010.

[15] Y. Wang, T. Feng, K. Wang, M. Qian, Y. Chen, and Z. Sun, "A facile method for preparing transparent, conductive, and paper-like silver nanowire films," Journal of Nanomaterials, vol. 2011, Article ID 935218, 5 pages, 2011.

[16] A. R. Madaria, A. Kumar, F. N. Ishikawa, and C. Zhou, "Uniform, highly conductive, and patterned transparent films of a percolating silver nanowire network on rigid and flexible substrates using a dry transfer technique," Nano Research, vol. 3, no. 8, pp. 564-573, 2010.

[17] A. R. Madaria, A. Kumar, and C. Zhou, "Large scale, highly conductive and patterned transparent films of silver nanowires on arbitrary substrates and their application in touch screens," Nanotechnology, vol. 22, no. 24, Article ID 245201, 2011.

[18] T. Akter and W. S. Kim, "Reversibly stretchable transparent conductive coatings of spray-deposited silver nanowires," ACS Applied Materials \& Interfaces, vol. 4, pp. 1855-1859, 2012.

[19] J. van de Groep, P. Spinelli, and A. Polman, "Transparent conducting silver nanowire networks," Nano Letters, vol. 12, no. 6, pp. 3138-3144, 2012.

[20] S. De, T. M. Higgins, P. E. Lyons et al., "Silver nanowire networks as flexible, transparent, conducting films: extremely high DC to optical conductivity ratios," ACS Nano, vol. 3, no. 7, pp. 1767-1774, 2009.

[21] F. S. F. Morgenstern, D. Kabra, S. Massip et al., "Ag-nanowire films coated with zno nanoparticles as a transparent electrode for solar cells," Applied Physics Letters, vol. 99, Article ID 183307, 2011.

[22] R. Zhu, C.-H. Chung, K. C. Cha et al., "Fused silver nanowires with metal oxide nanoparticles and organic polymers for highly transparent conductors," ACS Nano, vol. 5, no. 12, pp. 9877-9882, 2011.

[23] D. Azulai, T. Belenkova, H. Gilon, Z. Barkay, and G. Markovich, "Transparent metal nanowire thin films prepared in mesostructured templates," Nano Letters, vol. 9, no. 12, pp. 4246-4249, 2009.

[24] Z. Yu, L. Li, Q. Zhang, W. Hu, and Q. Pei, "Silver nanowirepolymer composite electrodes for efficient polymer solar cells," Advanced Materials, vol. 23, pp. 4453-4457, 2011.

[25] L. Li, Z. Yu, W. Hu, C. h. Chang, Q. Chen, and Q. Pei, "Efficient flexible phosphorescent polymer light-emitting diodes based on silver nanowirepolymer composite," Advanced Materials, vol. 23, pp. 5563-5567, 2011.

[26] X. Y. Zeng, Q. K. Zhang, R. M. Yu, and C. Z. Lu, "A new transparent conductor: silver nanowire film buried at the surface of a transparent polymer," Advanced Materials, vol. 22, no. 40, pp. 4484-4488, 2010.

[27] D.-S. Leem, A. Edwards, M. Faist, J. Nelson, D. D. C. Bradley, and J. C. de Mello, "Efficient organic solar cells with solutionprocessed silver nanowire electrodes," Advanced Materials, vol. 23, pp. 4371-4375, 2011.

[28] J. Y. Lee, S. T. Connor, Y. Cui, and P. Peumans, "Semitransparent organic photovoltaic cells with laminated top electrode," Nano Letters, vol. 10, no. 4, pp. 1276-1279, 2010.

[29] H. Tian, D. Xie, and Y. Yang, "Flexible, ultrathin, and transparent sound-emitting devices using silver nanowires film," Applied Physics Letters, vol. 99, Article ID 253507, 2011.

[30] Z. Zhang, X. Chen, H. Yang, H. Fu, and F. Xiao, "Electrically conductive adhesives with sintered silver nanowires," in Proceedings of the International Conference on Electronic Packaging Technology and High Density Packaging (ICEPT-HDP '09), pp. 834-837, August 2009.

[31] M. Dressel and G. Gruner, Electrodynamics of Solids: Optical Properties of Electrons in Matter, Cambridge University Press, Cambridge, UK, 2002.

[32] G. Haacke, "New figure of merit for transparent conductors," Journal of Applied Physics, vol. 47, no. 9, pp. 4086-4089, 1976. 

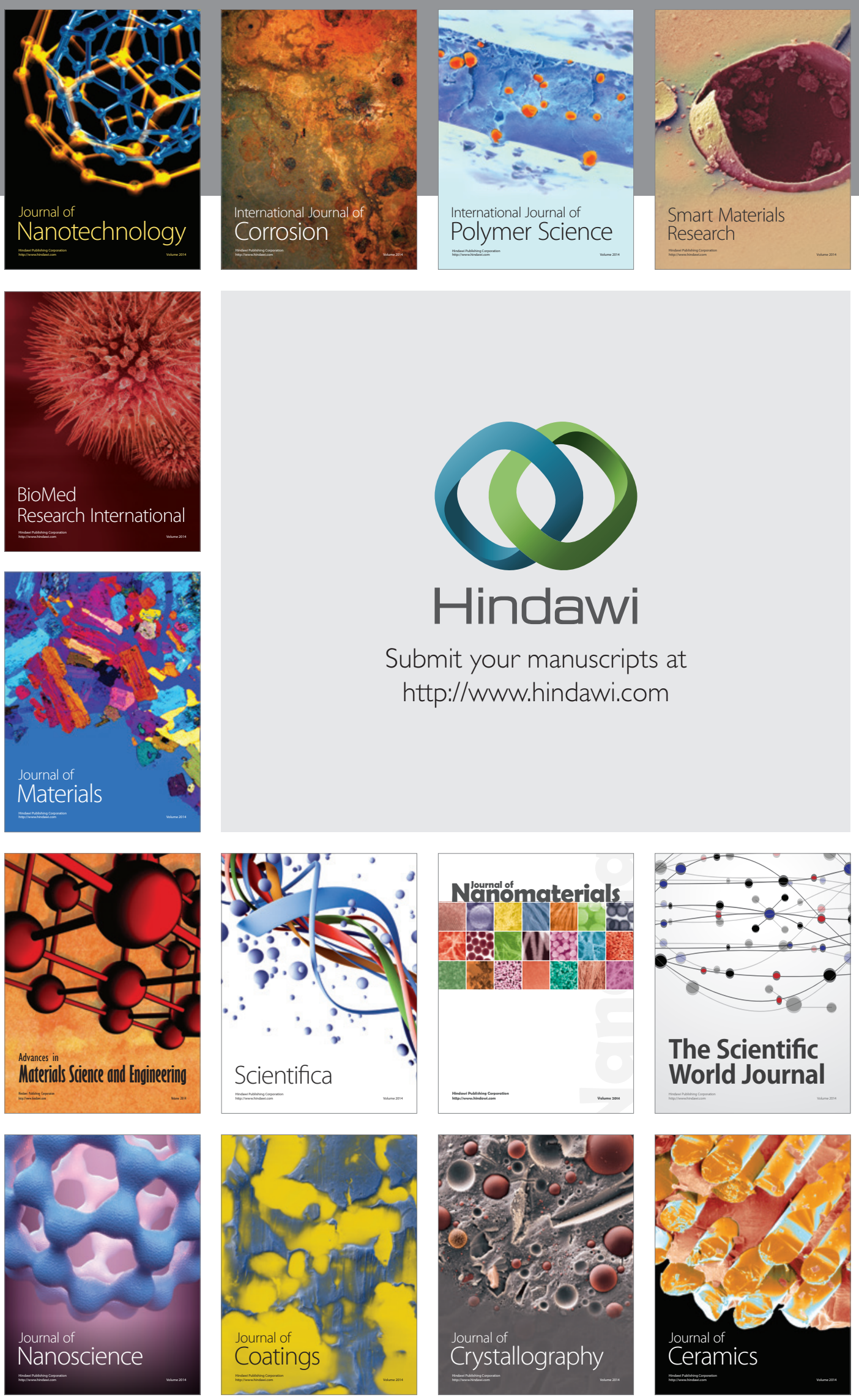

The Scientific World Journal

Submit your manuscripts at

http://www.hindawi.com

\section{World Journal}

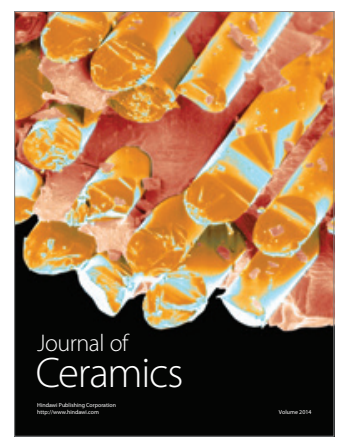

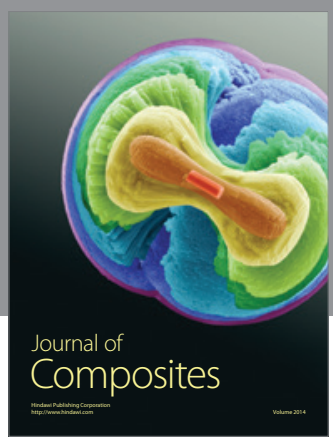
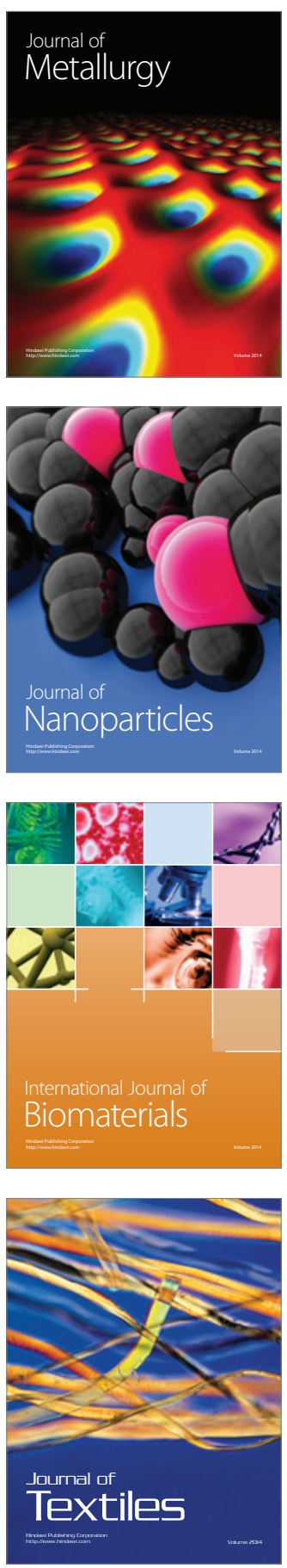